\title{
Dynamical nuclear decoupling of electron spins in molecular graphenoid radicals and biradicals
}

\author{
Federico Lombardi, ${ }^{1,}{ }^{*}$ William K. Myers, ${ }^{2}$ Ji Ma, ${ }^{3}$ Junzhi Liu, ${ }^{4}$ Xinliang Feng, ${ }^{3}$ and Lapo Bogani ${ }^{1}, \dagger$ \\ ${ }^{1}$ Department of Materials, University of Oxford, 16 Parks Road, Oxford, OX1 3PH, United Kingdom \\ ${ }^{2}$ Inorganic Chemistry, University of Oxford, South Parks Road, Oxford, OX1 3QR, United Kingdom \\ ${ }^{3}$ Centre for Advanced Electronics, Faculty of Chemistry and Food Chemistry, \\ Technische Universität, Monnsenstraße 4, 01069, Dresden, Germany \\ ${ }^{4}$ Department of Chemistry and State Key Laboratory of Synthetic Chemistry, \\ The University of Hong Kong, Pokfulam Road, Hong Kong, China.
}

(Dated: February 12, 2020)

\begin{abstract}
We investigate the mechanisms of nuclear decoupling in synthetically-tailored graphenenoids, where the electron spin state is introduced by topological manipulation of the lattice. We compare molecular graphenoids containing one and two spin centres, introduced by pentagonal rings in the honeycomb lattice. Exploiting the molecular nature of the systems, we investigate the role of different nuclear species and environments. Variations on the Carr-Purcell-Meiboom-Gill pulse trains are used to prolong the coherence time of the electron spin of the radicaloids, leading to substantial improvements in performance and coherence times up to $300 \mu \mathrm{s}$ at liquid nitrogen temperature. The investigation of electron spin coherence as a function of inter-pulse spacing, with times close to the inverse of the nuclear precession frequency, reveals that a train of pulses in-phase with the nuclear precession maximises the nuclear decoupling. At room temperature the limits imposed by the sample treatment and environment are reached, indicating what amelioration is necessary to further enhance the quantum performance.
\end{abstract}

\section{INTRODUCTION}

It is of paramount importance, for quantum applications, that the coherence time of a superposition of states is long enough to enable gate operations with sufficient fidelity [1]. As a consequence the decoherence mechanisms of quantum systems and ways to prevent them are currently under intense scrutiny [2].

Solid state spin quantum bits usually carry with them the presence of a nuclear environment [3]. How well this environment is defined, and whether it can actually be used to store and retrieve information, depends on the specific system under investigation. In some instances the nuclear environment can be a major source of decoherence, and where structural indetermination is present, this may produce considerable issues in the operation and standardization of the quantum bits. As a consequence, much attention is currently paid to ways of decoupling the nuclear spin bath from the electronic one [4-7] or, conversely, to the opportunities that can lie in carefully exploiting the nuclei-electron spin interactions [8-10]. These two seemingly different approaches are actually technically intimately connected: they both involve controlling the hyperfine coupling with an external train of impulses which will have the effect of polarise the nuclear spins and project their state onto the electron spin which is then measured [11].

The investigation of defects in solids has proved to be a very fertile ground for quantum materials, and impressive quantum properties have been recorded in nitrogenvacancy $(\mathrm{NV})$ centres in diamond [12] and in color cen-

\footnotetext{
* email: federico.lombardi@materials.ox.ac.uk

$\dagger$ email: lapo.bogani@materials.ox.ac.uk
}

tres in silicon carbide (SiC) [13]. Molecular systems have also produced increased enthusiasm in the last few years for their possible applications in the nano-region and the almost infinitive tunability offered by synthetic chemistry in terms of tailoring magnetic[14-16], optical[17] and electrical[18] properties of molecular groups. While initially it was thought that the quantum properties of molecular systems might be hampered by short coherence times [19], careful materials design using nuclear spin free ligands [20, 21], and exploitation of clock transitions [22$24]$ brought molecular systems as stable protagonists in the horizon of qubits candidates [25, 26].

Carbon nanomaterial are particularly interesting in this regard because of their conducting and mechanical properties, the very low spin-orbit coupling [27], the presence of edge states [28] and topologically-engineered states [29]. Although carbon nanotubes (CNTs) have shown low coherence times[30], of the order of ns at $\mathrm{mK}$ temperature, $T$, we recently reported $\mu$ s coherence in synthetically-tailored graphenoids, where pentagonal defects can be introduced at precise positions [31]. These systems allow studying the effects of topology on the quantum properties of graphene, as pentagonal sites introduce a spin triplet component into the electronic wavefunction. Superior quantum coherence at nitrogen $T$ is obtained, and up to $2 \mu \mathrm{s}$ coherence time in solution is reached at room $T$.

One way of overcoming the issue of nuclei-induced decoherence is to engineer the material using isotopic enrichment, cutting down the fraction of spin-containing nuclei [32]. It is anyway desirable to develop new methods to prolong the electron spin coherence without having to introduce ultra-pure environments: not only does this eliminate a difficult and expensive material engineering step, but it may also widen the range of envi- 
ronments where the quantum centre can be operated. To this aim nucleus-electron spin-spin decoupling can be employed [4], e.g. by using the Carr-Purcell-MeiboomGill (CPMG) sequence of pulses [33, 34]. CPMG was originally developed for structural nuclear magnetic resonance studies, but its applicability to the enhancement of quantum units is recognized.

Here we detail the effect of CPMG pulse sequences on the electron spin coherence of molecularly-made graphenoids in frozen solutions at $100 \mathrm{~K}$. We briefly introduce the quantum system, we explore its unoptimised coherence time and then proceed enhancing it with dynamical decoupling. We show that we can selectively decouple a specific nucleus by tailoring the time interval between pulses in a CPMG sequence and we discuss the limits and advantages of this approach.

\section{EXPERIMENTS AND ANALYSIS}

The graphenoids explored in this study are produced with a bottom-up approach[31]. Using an organic synthetic approach[35-39] it is possible to create aromatic molecular systems that contain 16 hexagonal rings and two pentagonal rings placed with atomic precision, so as to create an available open-shells ground state in each molecule and spin-bearing centres, as depicted in Figure 1a. The resulting molecular graphenoids contain one or more spin centres, depending on the hydrogenation of the rings. Monoradical and biradical molecular species are sometimes mixed, in the compounds, with a molar fraction of monoradical of $\simeq 0.02[31]$.

The spin properties of the system are described by the following Hamiltonian[31]:

$$
\mathcal{H}_{0}=\mu_{\mathrm{B}}\left(S_{1}+S_{2}\right) g H+S_{1} J S_{2}+\sum_{j=1}^{N} S A_{j} I_{j} .
$$

where $H$ is the external magnetic field, $\mu_{\mathrm{B}}$ is the Bohr magneton, $g$ the electron Landé factor, $J$ is the exchange interaction between the electronic spins $S_{1}$ and $S_{2}$, the $A_{j}$ parameters describe the hyperfine interaction with the nuclear spins $I_{j}$ and the sum runs over all $N$ interacting nuclei (both intra- and inter-molecular ones, and including carbon, hydrogen and deuterium atoms). As previously reported [31], the exchange interaction $J \sim-550$ $\mathrm{K}$ and the molecules are in an antiferromagnetic ground state, with a closely lying ferromagnetic excited state that can be populated increasing $T$. Because the biradical is coupled anti-ferromagnetically, and the monoradical is present in a fraction of a few $\%$, the monoradical species dominates the magnetic signal at $T \leq 80 \mathrm{~K}$. These monoradial molecules can be modelled using the same Hamiltonian of Eq.1, simply ignoring one of the electron spins. As previously reported [31], the molecular fraction $\alpha \sim 2 \%$ is estimated from the $T$-dependence of the EPR signal and magnetization measurements.

The samples were tested by preparing three solutions with a concentration of $1.5 \pm 0.5 \mathrm{mM}$ : in toluene, deuterated toluene ( $d$-toluene), and carbon disulfide $\left(\mathrm{CS}_{2}\right)$.

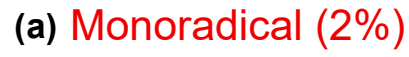

Biradical

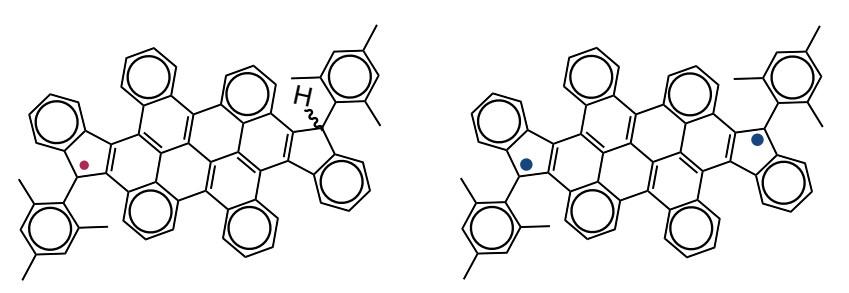

(b)

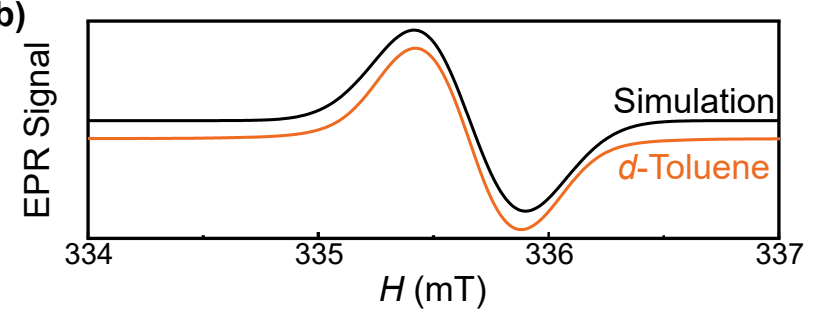

FIG. 1. (a) Chemical structure of the monoradical (left) and the biradical (right) graphenoids studied here. Hydrogen atoms are omitted, except for the one that is responsible for the different mono-bi-radical character owing to incomplete dehydrogenation. (b) Continuous wave EPR signal measured at room temperature at $9.40 \mathrm{GHz}$ for a $d$-toluene solution (orange) and simulation with the Hamiltonian in Eq.1 (black).

These three solvents offer platforms with different hyperfine baths: toluene contains 8 hydrogen atoms and 7 carbons with the natural concentration of ${ }^{13} \mathrm{C}$; deuteration decreases the hyperfine contribution of hydrogens substituting them with weaker deuterium; eventually $\mathrm{CS}_{2}$ contains only the natural percentage of ${ }^{13} \mathrm{C}$. In this way both the effect of nuclei in the environment and inside the molecule itself can be probed.

We measure the coherence times in ensembles through pulse Electron Paramagnetic Resonance (EPR) [40]. In EPR experiments the spin levels are split by the application of the static magnetic field $H$, and the spin transitions are excited using microwave irradiation inside a microwave resonator of relatively low quality factor $Q \simeq 300$. In the following we exclusively operate at $\mathrm{X}$-band, i.e. at $9.40 \mathrm{GHz}$. We carried out the experiments in a Bruker Elexsys E580 equipped with a $1 \mathrm{~kW}$ travelling wave tube amplifier and the resonator Bruker ER4118X-MS-3W1. T was regulated by an Oxford Instruments CF9350 cryostat with ITC503. Simulation of the static EPR spectra was performed using Eq. 1 and the Matlab package Easyspin [41]. Good agreement is obtained with $g=2.0027 \pm 0.0005$, while $A_{j}$ were not resolved in this type of measurement (Figure 1b).

The evolution of the spin wavefunction contains a part that sees momentum exchange with the lattice (i.e. will produce variations of the $S_{z}$ component of the spin) and a part that only produces a variation in the phase of the wavefunction, i.e. that contains most of the quantum information. This quantum evolution of a spin is often visualized as a movement over the Bloch sphere, as in Figure 2: zenith positions indicate pure $|\uparrow\rangle$ and $|\downarrow\rangle$ states, and any possible quantum state 
(a)

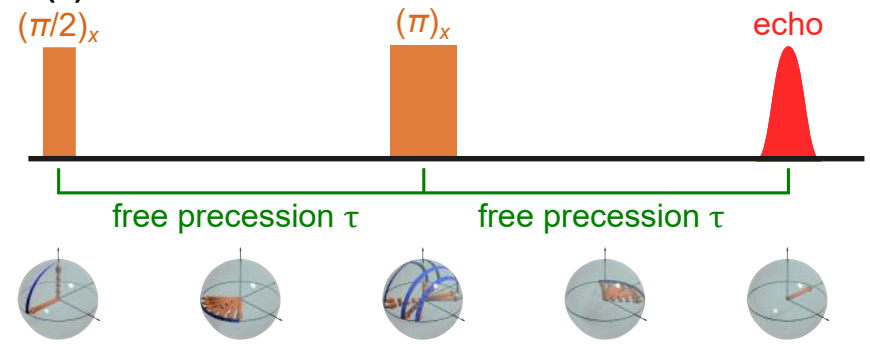

(b)

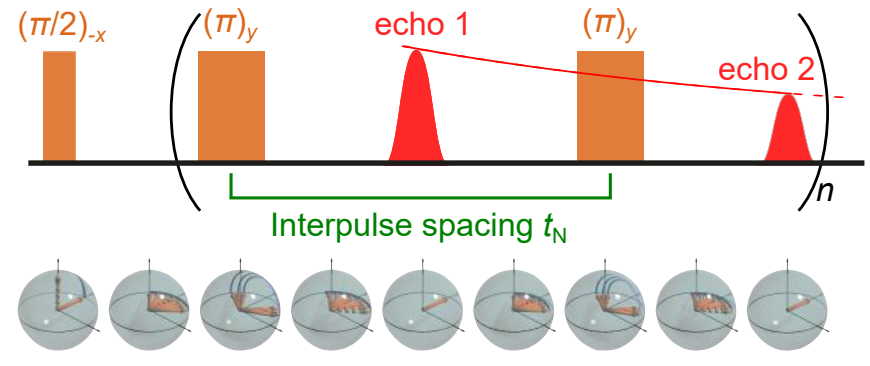

(c)

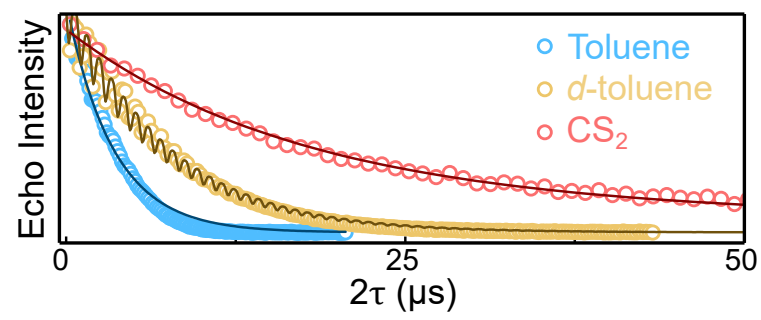

FIG. 2. (a) Pulse sequence used for the detection of the Hahn echo and effect of the pulses on the Bloch sphere. (b) Pulse sequence used for the detection of coherence with decoupling from the nuclear bath by CPMG. (c) Decay of the Hahn echo signal, as recorded in different solvents: toluene (blue), deuterated toluene (yellow) and $\mathrm{CS}_{2}$ (red). Lines are fits to the data with Eq. 2. All measurements are performed at $9.40 \mathrm{GHz}$ and $T=100 \mathrm{~K}$.

$|\psi\rangle=\cos \theta / 2|\uparrow\rangle+e^{\mathrm{i} \phi} \sin \theta / 2|\downarrow\rangle$ is represented by a point on the spherical surface. The decay times of the two parts are defined as $T_{1}$ (representing vertical displacement on the Bloch sphere or variations of $\theta$ ) and $T_{2}$ (evolution of the quantum phase $\phi$, described by the azimuthal movement), which are also called spin-lattice and coherence times, respectively. In the literature several notations and definitions of the coherence time can be retrieved. Here, we refer to the coherence time as $T_{\mathrm{m}}$, and by this we consider a time which encompasses the contribution of $T_{2}$ and other effects such as instantaneous and spectral diffusion, residual dipolar interactions in dilution, and orientational averaging [40] so that, in general, $T_{\mathrm{m}} \leq T_{2}$.

Measurements that fully include the effect of the nuclear bath, i.e. without any attempt at nuclei-electron spin decoupling, are acquired using an Hahn-echo sequence (Figure 2a). The experiment is performed by using a microwave $\pi / 2$ pulse resonant with the spin transitions applied along the $x$ axis of the rotating frame. In this way the molecular spin state is transformed into the superposition state $|\Psi\rangle=(|\uparrow\rangle+|\downarrow\rangle) / \sqrt{2}$. To better describe the effect of the pulses we can use the propagator formalism. During the pulse the total Hamiltonian of the system is $\mathcal{H}=\mathcal{H}_{0}+\omega_{1} S_{x}$ and the evolution operator can be written as $U=\exp \left\{-\mathrm{i} \int \mathcal{H} \mathrm{d} t\right\}$. In the free precession the Hamiltonian is only $\mathcal{H}_{0}$ with propagator operator $U=e^{\mathrm{i} \mathcal{H}_{0} t}$. This means that when brought in the $x y$ plane the different spin packets having different resonance frequencies $\omega_{i}$ will accumulate a phase difference $\Delta \phi=\left(\omega_{i}-\omega_{\mathrm{mw}}\right) \tau$. The phases are then refocused by the $\pi$ pulse and detected as an echo. The decay of the echo signal on changing the free precession time is depicted in Figure 2c for all the solvents. We fit the decay of echo signal, $Y(t)$, as a function of time $t$ with:

$$
Y(t)=Y_{0}\left[\xi_{1} \exp \left(\frac{-t}{T_{\mathrm{m}}^{\text {mono }}}\right)+\xi_{2} \exp \left(\frac{-t}{T_{\mathrm{m}}^{\mathrm{bi}}}\right)\right] \Xi(t),
$$

where the coherence times of the monoradical and biradical species are labelled $T_{\mathrm{m}}^{\mathrm{mono}}$ and $T_{\mathrm{m}}^{\mathrm{bi}}$ and the coefficients $\xi_{i}$ are their respective weights.

$\Xi(t)=1+\left[k_{1} \sin \left(2 \omega \tau+\phi_{1}\right)+k_{2} \sin \left(4 \omega \tau+\phi_{2}\right)\right]$ introduces a signal modulation given by the hyperfine coupling, at a nucleus-specific frequency. The frequency of the nuclear precession is the Larmor frequency $\omega=\gamma_{i} H$, where $\gamma_{i}$ is the gyromagnetic ratio of the specific nucleus. Given the excitation frequency used, and considering that it can vary slightly in the set up of one experiment to another, we obtain the resonant field: $H \sim 340-$ $350 \mathrm{mT}$. This field corresponds to Larmor frequencies $\omega / 2 \pi=14.8 \mathrm{MHz}$ for ${ }^{1} \mathrm{H}$ and $2.4 \mathrm{MHz}$ for ${ }^{2} \mathrm{H}$, with amplitudes $k_{1}$ and $k_{2}$, and phases $\phi_{1}$ and $\phi_{2}$ for first- and second-order effects. Because the frequency is nuclearspecific, it can actually be used as a fingerprint of the dominant nuclear effects but, in the Hahn echo fitting procedure, the $\Xi$ term does not directly change the extraction of the $T_{\mathrm{m}}^{(i)}$. Hyperfine interactions are responsible for part of the decoherence but, for the purposes of fitting the echo decay signal and extracting $T_{\mathrm{m}}^{(i)}$, the nuclear modulation and the electron spin coherence decay act as two distinct parts of the fitting function: the $T_{\mathrm{m}}^{(i)}$ free parameters are independent of $\Xi$, and $\Xi$ only provides information of the nuclear species that are creating signal oscillations.

The weights $\xi_{i}$ in Eq.2 are determined by the singlettriplet energy splitting $|J|=550 \mathrm{~K}$ and the monoradical molecular fraction $\alpha \sim 2 \%$ :

$$
\xi_{2}=\frac{e^{-J / k_{\mathrm{B}} T}}{\alpha+e^{-J / k_{\mathrm{B}} T}}, \quad \xi_{1}=1-\xi_{2} .
$$

Error analysis shows that the largest error contribution arises from uncertainties in the values of $\xi_{i}$. We considered a variation of $\pm 20 \%$ in the value of $\xi_{2}$ and fitted the experimental traces with the three pairs of $\xi_{i}$ obtained. We then extrapolated the error as the maximum difference between the two extreme values of $\xi_{i}$ and the central values. For all the solvents, we obtained that a $20 \%$ variation of $\xi_{2}$ produced about the same error on $T_{\mathrm{m}}^{\mathrm{bi}}$, and this quantity was plotted as error bars. 
The nuclear modulation of the decay is visibly different, and with different $\omega$ for the three solvents. The $k_{1}$ and $k_{2}$ are usually very small for hydrogen and the modulation can only be seen for high sampling frequency which, conversely, is not ideal if you want to observe a long decay, therefore modulation effects are not well represented in Figure 2c for toluene. On the other hand, modulation is very clear for the deuterium in $d$-toluene that has large modulation depth $k_{1}$ and $k_{2}$ and low frequency. In the case of $d$-toluene we could expect to observe the effect of the intramolecular hydrogens, however their modulation is completely overshadowed by the deuterium and cannot be resolved in this type of measurement despite the two contributions being at two different frequencies. Similarly, for $\mathrm{CS}_{2}$ no modulation is observed because the solvent gives no nuclear contribution and the intramolecular hydrogens are too weak to produce a modulation in this type of measurement.

Having observed that nucelar modulation of the decay is an important factor in the Hahn echo, we now investigate dynamical decoupling as a way to increase robustness of electron coherence against nuclear hyperfine noise. Multiple schemes have been suggested [33, 34, 42-45], e.g. periodic and concatenated dynamical decoupling. In periodic dynamical decoupling the train of pulses are spaced of the same interval, and the CPMG decoupling scheme belongs to this family (Figure $2 \mathrm{~b}$ ). In concatenated dynamical decoupling, the pulses are optimised to be at specific time and the interpulse spacing is different between adjacent decoupling pulses: Uhrig Dynamical Decoupling (UDD) is one example [44]. The theory predicts that in case of an environmental bath with a hard cut-off spectral density [3], UDD performs better than CPMG, while in the presence of a soft cut-off they have comparable efficiency [46]. However, it is often hard to make theoretical predictions without a specific reference to a quantum system and CPMG outperforms UDD when the bath is composed of weakly-coupled nuclei [47, 48].

We employed a two-step phase cycling on the initial pulse to eliminate pulse offset effects [40, 48]. Each CPMG measurement produced a set of echoes, the maximum of each echo was fitted with the model in Eq.2 where no modulation is expected (as depicted in Figure $2 \mathrm{~b}$ ). The trend of the signal can then be followed on varying the inter-pulse spacing, $t_{\mathrm{N}}$, between the $\pi$ pulses of the CPMG train (Figure $2 \mathrm{~b}$ ): for each $t_{\mathrm{N}}$ value one decay trace is obtained, from which we extract the two decay constants $T_{\mathrm{CPMG}}^{\mathrm{mono}}$ and $T_{\mathrm{CPMG}}^{\mathrm{bi}}$, for the monoradical and biradical species respectively. We compare the results with the precession periods of the nuclei involved: hydrogen, deuterium and carbon. The precession period is just the inverse of the Larmor frequency and in our experimental conditions we obtain: $T_{\mathrm{H}} \simeq 70 \mathrm{~ns}, T_{\mathrm{D}} \simeq 457$ ns, and $T_{\mathrm{C}} \simeq 280 \mathrm{~ns}$ (for ${ }^{13} \mathrm{C}$ ).

Figure 3 displays the resulting behaviour of the $T_{\mathrm{CPMG}}^{(i)}$ when varying the inter-pulse spacing in toluene. The signal is mainly modulated by the precession of the ${ }^{1} \mathrm{H}$ nuclei, and decays rapidly on increasing the interpulse spacing as predicted by theory [49]. This is to be assigned to

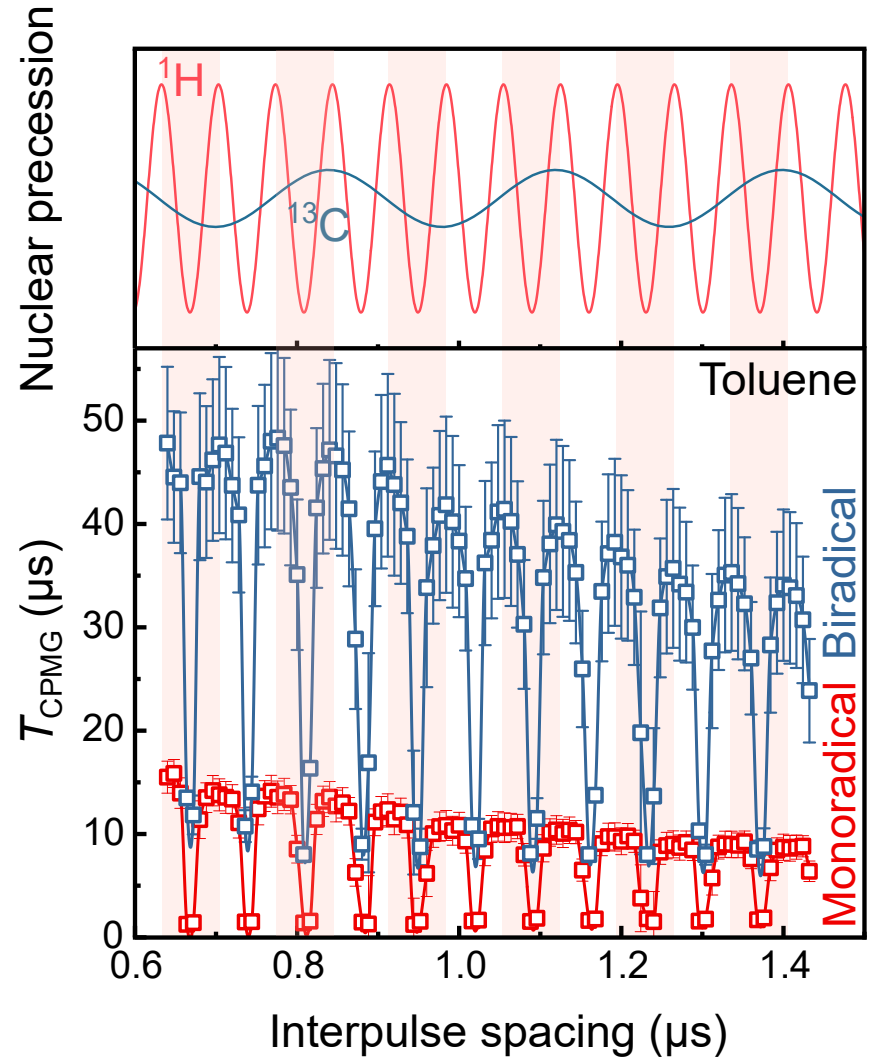

FIG. 3. Top panel: Periodic oscillations of the nuclear signal of an ensemble, as produced by precession, for ${ }^{1} H$ hydrogen (red) and a ${ }^{13} C$ (blue) atoms. Bottom panel: experimental dependence of the decay time $T_{\mathrm{CPMG}}$ as a function of the interpulse spacing interval $t_{\mathrm{N}}$. The signals for the monoradical and biradical species are plotted in red and blue, respectively. In both panels the periodicity of the hydrogen nuclei is highlighted by red shading. All measurements were acquired in toluene at $T=100 \mathrm{~K}$.

both intra- and inter-molecular ${ }^{1} \mathrm{H}$ atoms, as the toluene solvent molecules are capable of $\pi$-stacking onto the aromatic surface of the graphenoids. In Figure 3 it is also clear how the efficiency of the decoupling technique decreases when increasing the inter-pulse spacing. If the spacing is large, the spins are allowed to decohere between one pulse and the next one and the effect of the decoupling becomes less evident. Ultimately, how fast this happens depends on the unoptimised value of $T_{\mathrm{m}}$ measured through the Hahn echo sequence: when the inter-pulse spacing $t_{\mathrm{N}} \simeq T_{\mathrm{m}}$, the CPMG decoupling will not be effective.

The maximum decoupling is obtained when the interpulse spacing is equal to integer multiple of the precession period of the targeted nucleus. In other words, if the train of refocusing pulses is perfectly in phase with the precession of the nuclei, the quantum phase becomes more robust against the decohering effect of the nuclei themselves. For both mono and bi-radical the $T_{\mathrm{CPMG}}^{(i)}$ can reach, in the in-phase scenario, up to 5 times larger than if the pulse sequence is out-of-phase with the nuclear precession. This is a non-negligible improvement 


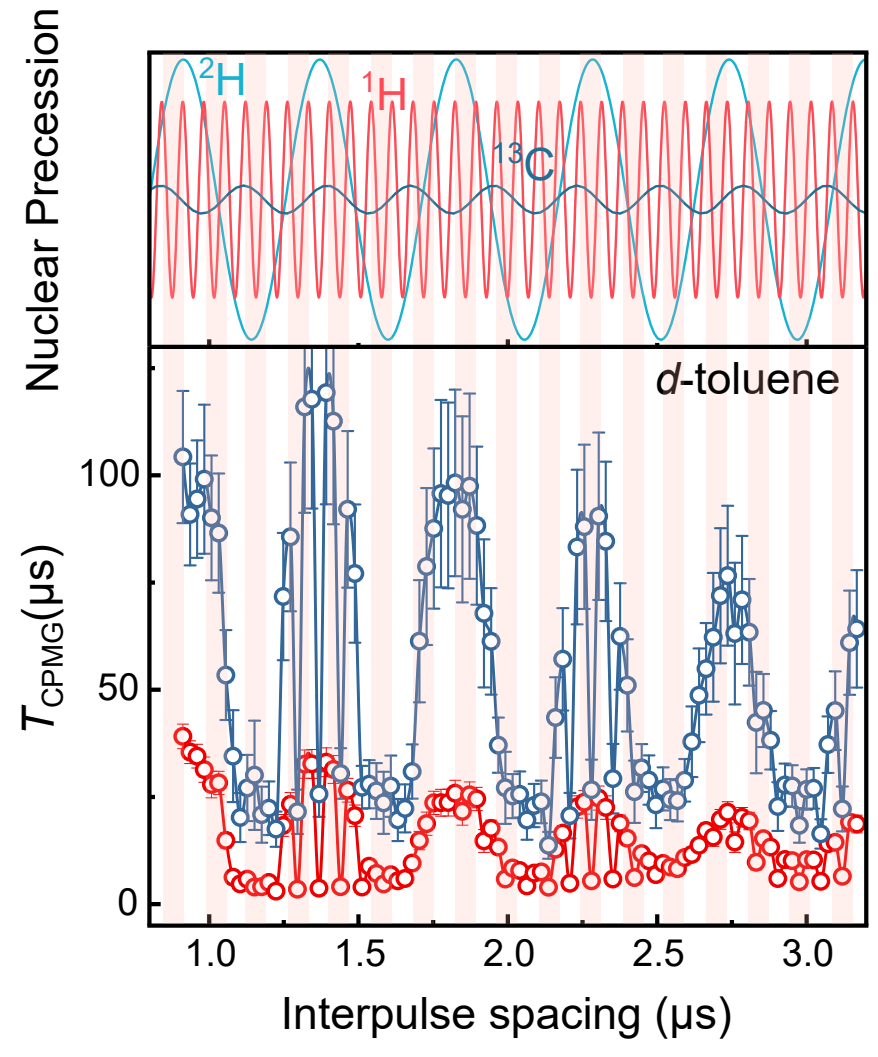

FIG. 4. Top panel: Periodic oscillations of the nuclear signal of an ensemble, as produced by precession, for ${ }^{2} D$ deuterium (teal), ${ }^{1} H$ hydrogen (red) and a ${ }^{13} C$ (blue) atoms. Bottom panel: experimental dependence of the decay time $T_{\mathrm{CPMG}}$ as a function of the interpulse spacing interval $t_{\mathrm{N}}$. The signals for the monoradical and biradical species are plotted in red and blue, respectively. In both panels the periodicity of the hydrogen nuclei is highlighted by red shading. All measurements were acquired in deuterated toluene at $T=100 \mathrm{~K}$.

over the

The behaviour in $d$-toluene, shown in Figure 4 , displays $T_{\mathrm{CPMG}}^{(i)}$ modulated by both by the ${ }^{1} \mathrm{H}$ and ${ }^{2} \mathrm{H}$ nuclei. Here, it is relevant to highlight the effect of the ${ }^{1} H$ nuclei. Indeed, above we stated that their modulation was not detected in the simple Hahn Echo experiment. However, when using CPMG the effect is distinguishable and the modulation depth is analogous to the one produced to the one of the ${ }^{2} H$ nuclei. This result needs to be included in a wider picture that encompasses the possibility of detecting very weakly coupled nuclei with dynamical decoupling techniques. This was proved in the literature in the case of NV centres [11]. And in this case a carefully understanding of the dynamics of the decoupling could pave the way to electron to nuclear coherence transfer and storing of the quantum phase on the nuclear coherence.

Finally in Figure 5 we note that in $\mathrm{CS}_{2}$ hydrogens are again the main modulating factor. Similarly to the $d$-toluene case, a very strong modulation now appears which is a fingerprint of very weakly coupled intermolecular hydrogens. Significantly, $T_{\mathrm{CPMG}}^{\mathrm{bi}}$ initially increases

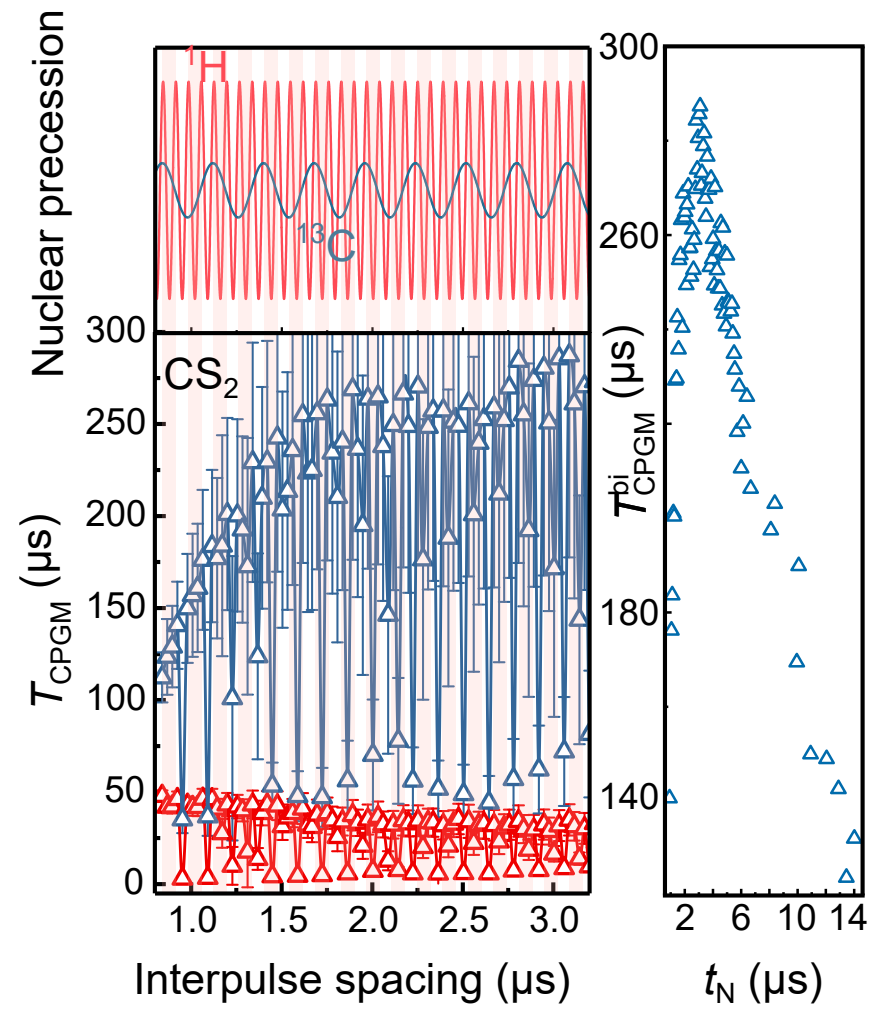

FIG. 5. Left panel: Periodic oscillations of the nuclear signal of an ensemble, as produced by precession, for ${ }^{1} H$ hydrogen (red) and a ${ }^{13} C$ (blue) atoms and experimental dependence of the decay time $T_{\mathrm{CPMG}}$ as a function of the interpulse spacing interval $t_{\mathrm{N}}$. The signals for the monoradical and biradical species are plotted in red and blue, respectively. The periodicity of the hydrogen nuclei is highlighted by red shading. All measurements were acquired in carbon disulfide at $T=100$ K. Right panel: $T_{\mathrm{CPMG}}^{\mathrm{bi}}$ decay versus $t_{\mathrm{N}}$, for simplicity only the maximum peaks over a period are shown and errorbars are removed.

when increasing the interpulse spacing, contrary to the behaviour in toluene and $d$-toluene. However, if we track the $T_{\mathrm{CPMG}}^{(i)}$ for long $t_{\mathrm{N}}$ up to $14 \mu \mathrm{s}$ (Figure $5 \mathrm{~b}$ ) we find again the decay that we attributed to the efficiency of the decoupling sequence. We explain the initial rise considering that the decay time observed in $\mathrm{CS}_{2}$ is much larger than the $T_{\mathrm{CPMG}}^{(i)}$ in the other solvents and, accordingly, the number of pulses required to map it is also considerably larger: up to 400 pulses are required. In this case, imperfections in the pulse shaping should also be considered: there is a systematic error in pulse formation that accumulates when in presence of many pulses [50]. Pulse errors can thus become the main source of decoherence, limiting the benefits of a dynamical decoupling approach [51].

We evaluate the overall performances of the dynamical decoupling scheme in Figure 6, by plotting together the Hahn Echo decays, the CPMG traces for the three solvents and the $T_{1}$ time, which is an upper limit to the coherence. Only the $T_{1}$ measured in $d$-toluene is depicted, because the spin-lattice relaxation time is independent 


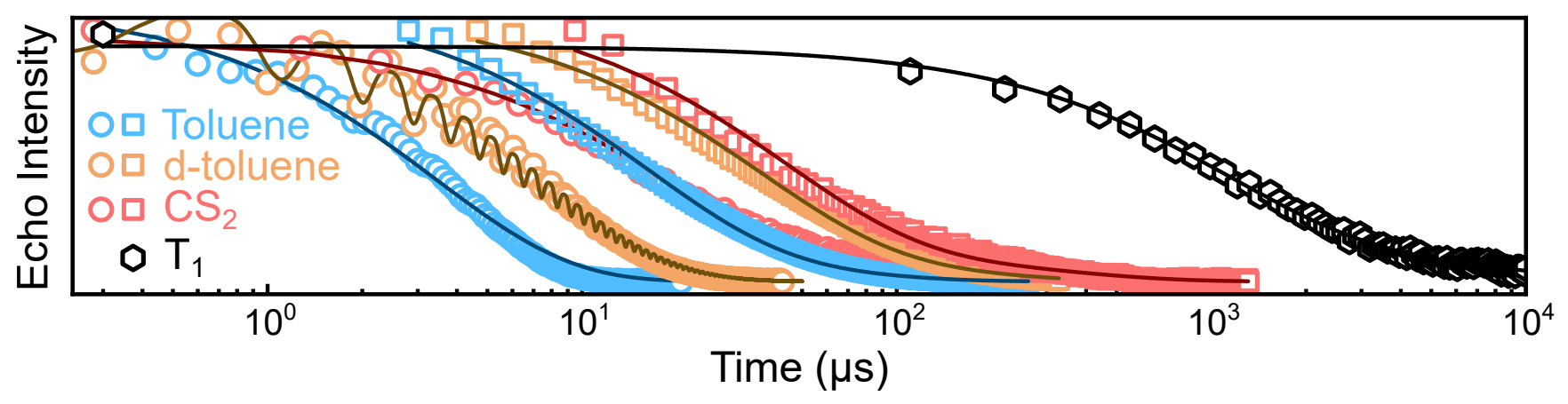

FIG. 6. Echo signal intensity as a function of the distance between the echoes and the first impulse $\pi / 2$ for the Hahn echo (circles) and CPMG sequence (squares). Measurements are performed in toluene (turquoise), $d$-toulene (amber) and carbon disulfide (magenta). The spin-lattice relaxation time $T_{1}$ is also reported (black hexagons) as measured via a signal inversion recovery procedure, here displayed as a decay for simplicity. All measurements are performed at $9.40 \mathrm{GHz}$ and $T=100 \mathrm{~K}$.

from hyperfine coupling and produces a similar result in all three solvents. When compared to the respective Hahn echo coherence times and using the best interpulse spacing, we observe a more-than-ten-fold increase by decoupling in toluene, a six-fold increase in $d$-toluene and a three-fold increase inc $\mathrm{CS}_{2}$. The most important relative improvement is obtained in toluene because it has the largest number of environmental nuclei, while smallest improvement is obtained in the system with the best performance, $\mathrm{CS}_{2}$, where very few environmental nuclei matter. In $\mathrm{CS}_{2}$ we reach coherence time of $0.3 \mathrm{~ms}$, against a $T_{1}$ of $2 \mathrm{~ms}$, while the maximum value attainable in theory would be $2 T_{1}$. This theoretical limit is not reached because the decoupling can only mitigate the effect of the nuclei but cannot erase it completely. Were this the case, the coherence time in toluene after CPMG should also be the same of $\mathrm{CS}_{2}$ without CPMG.

We eventually wish to stress an often-overlooked aspect of dynamical decoupling: the presence of additional signals that arise when having multiple pulses. In EPR, for any three-pulse sequence, one should always observe a stimulated echo [40]. If the time distance between the pulses is the same, as in periodic dynamical decoupling, it is possible that the stimulated echo overlaps with the original echo signal, distorting the signal shape (Figure 7a) and possibly mixing the $T_{m}$ and $T_{1}$ times. In the sequence of Figure 2 we used a 2-step phase cycling: the first step has the $\pi / 2$ pulse with phase $180^{\circ}$ and the second step with phase $0^{\circ}$, so that all $\pi$ pulses are applied on the $y$ axis and their relative phase is always kept constant. In the context of the analysis of quantum systems, this choice is justified by the fact that the resulting time is still the time in which the quantum bit system can be operated meaningfully, i.e. it corresponds to the desired quantity. On the other hand, it is instructive to understand how the overlap is affecting the results, by using different phase cycling techniques that minimize it. To completely eliminate unwanted signals we would need a phase-cycling with $4^{n-1}$ steps, where $n$ is the number of $\pi$ pulses[52], which would mean an absurdly-large pulse sequence, in the present case. We thus test the difference between the simple 2-phase step cycling and a more complex scheme employing 8 phases, as depicted in Figure $7 \mathrm{~b}$. In this more sophisticated phase cycling we still cycle the first $\pi / 2$ pulse, but we also vary the phase difference among the following $8(\pi)_{y}$ pulses. In Figure 7a we define $\varphi_{i}$ the phase difference between two adjacent $(\pi)_{y}$ pulses. In each step of the scheme $\varphi_{i}$ can assume the value $0^{\circ}$ or $180^{\circ}$, which implies having $(\pi)_{y}$ or $(\pi)_{-y}$. In order to cancel the overlap of the signals the sequence is cycled over all the $2 \times 2^{7}$ possible different combinations, for a total of 256 steps.

The two approaches were tested at room temperature. In Figure 7c we display the difference in the decay of the signal when the two different cycling sequences are employed. A discrepancy in the decay is clearly visible, with the 256-step measurement relaxing faster than the 2 -step one. Additionally, in the 256-step measurement all the 8 echoes assume an almost-ideal shape (Figure 7a), while the stimulated echo distorts the shape of the higher order echoes in the 2-step phase cycling.

The variation of the results between the two different phase cycling can be attributed, once again, to pulse formation errors. The $\pi$ pulses in the train are supposed to refocus the phase of the different spin packets, by rotating them in the $x y$ plane. This is what is depicted in the Bloch sphere representation of Figure 2. However, because the rotation angle of a pulse is subjected to errors, the spin packets can be brought outside of the $x y$ plane, thus gaining a small but non-negligible $z$ component. Using a scheme that cycles the train of $\pi$ pulses between $y$ and $-y$ compensates for pulse formation errors and mitigates the formation of a vertical displacement. When discussing the time decay $T_{\mathrm{CPMG}}$ for standard 2step phase cycling it is useful to point out that this might contain an admixture of $T_{\mathrm{m}}$ and $T_{1}$, because the vertical displacement decays following $T_{1}$.

\section{CONCLUSIONS}

In conclusion, our measurements show in detail how CPMG sequences can be used to enhance the coherence time of molecular graphenoids, and how the sequences 

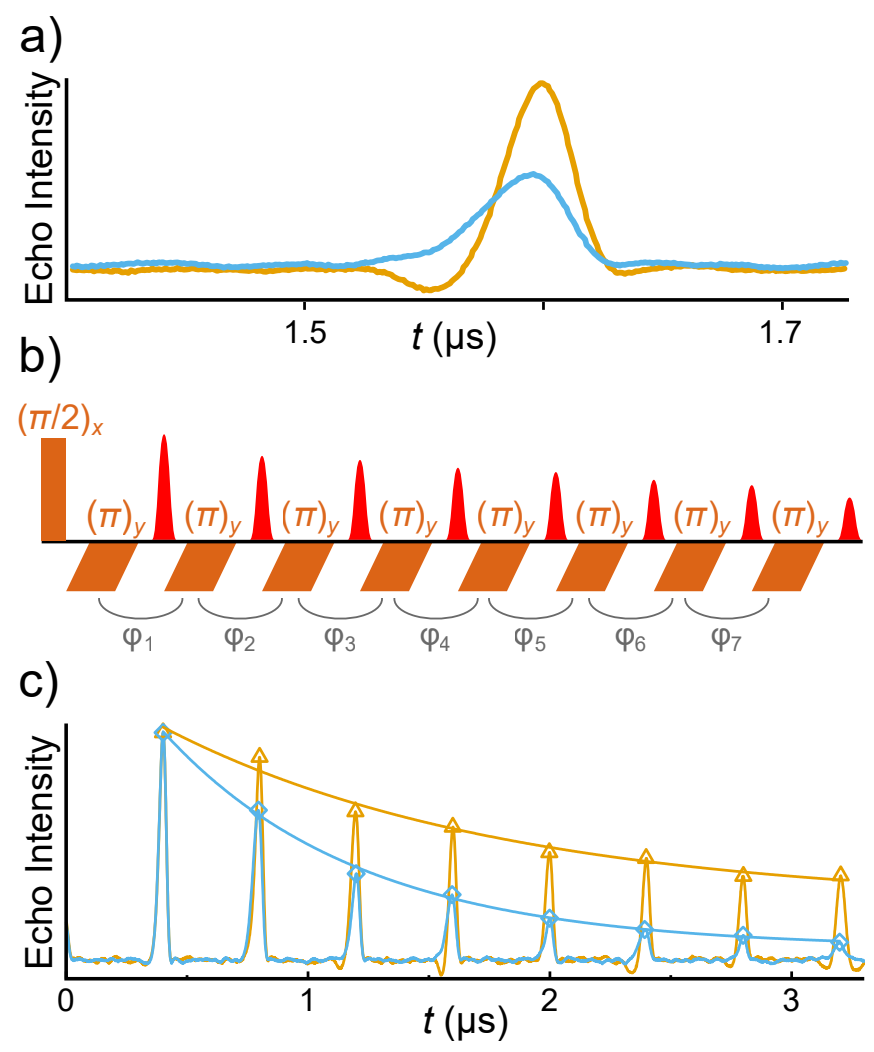

FIG. 7. a) Distortion of the echo signal produced by the overlap of multiple echoes, in the 2-step phase cycling of the CPMG sequence (orange). The equivalent echo signal after 8-phase cycling is also depicted (blue). b) Pulse sequence for 8-phase cycling in CPMG: after the initial $\pi / 2$ step, the next $8 \pi_{y}$ pulses have a $\varphi_{i}$ phase that cyclically assumes a value $0^{\circ}$ or $180^{\circ}$. c) Comparison of the echo decays obtained in CPMG with 2-step phase cycling and the 256-step one. Black lines are single exponential fits of the maximum of the echoes. All measurements are performed at room temperature.

can be tailored depending on the specific environment of the molecules, so as to optimize their quantum performance. The main source of decoherence in frozen solutions in the temperature range $80-150 \mathrm{~K}$ comes from the hyperfine contribution of the nuclei in the solvent and in the molecules: hydrogen and carbon atoms. The investigation of the effect of solvents highlights the important role played by the environment and the possibility of increasing the coherence times by addressing nuclei contained in the solvent molecules, instead of the molecular ones. The inclusion into environments that have very low content of active nuclei, thus reducing the solvent nuclear bath, allowed decoupling selectively from the molecular hydrogens. We regard that the hydrogens are the nuclei contributing the most to the decoherence, and indeed their modulation can be clearly seen independently of the solvent. Therefore, isotopical substitution of the hydrogens in the molecules could produce a significant increase of the coherence time. In fact, even when maximally decoupled, the coherence time in toluene is still half the one of decoupled $d$-toluene. On the other hand, there is no strong evidence of modulation of ${ }^{13} \mathrm{C}$ even in $\mathrm{CS}_{2}$. Due to the very low abundance of the isotope we are unable to detect its effect, although it could become more relevant in planned single-molecule measurements.

In addition we have explored possible sources of errors, with particular attention to the possibility of residual $T_{1}$ components in the $T_{\mathrm{CPMG}}$. While such tests are important on the methodological level, it is fundamental to stress that, whilst it is possible to achieve a $2^{n}$ step phase cycling for 8 pulses, this becomes more challenging when increasing the number of pulses, and already for 20 pulses the scheme would require $10^{6}$ which is technologically infeasible.

On a different note, our results indicate that the coherence time could still be improved upon by technical improvement in the experimental setup. First of all, a larger bandwidth resonator would allow minimising the interpulse spacing to the time of a single revolution of hydrogens that in this condition we found $T_{\mathrm{H}} \simeq 70 \mathrm{~ns}$. Second, to make up for the large amount of pulses required, a possible perspective is to implement additional correction schemes. One option is to introduce and compare other decoupling sequences, such as those tested for other quantum units $[45,47,53-59]$. Another possible source of inspiration arises from the efforts to correct pulse-formation errors produced in nuclear magnetic resonance, albeit for different instrumentation, and how if can be adapted to EPR in this particular situation. In theory, it would be possible to reduce the errors either using composite pulses [60-62] or shaped pulses [63]. Careful studies will anyway need to consider that this comes at the expense, in both cases, of an increase in the pulse duration time, therefore slower spin manipulation. Additionally, it would be possible to use, so called, self correcting sequences. These are dynamical decoupling sequences that employ rotations around multiple axes and are expected to correct pulse formation errors. One example is the XY4 sequence that was developed for NMR as an upgrade of CPMG [64]. This scheme has seen interesting theoretical developments in the last few years and experimental proof of better performances than CPMG $[45,47,58,59]$. Future studies of dynamical decoupling in molecular systems should incorporate the XY paradigm.

At room temperature the resulting decoherence is no longer dominated by the nuclear bath, as the limit of $T_{\mathrm{m}} \simeq 2 T_{1}$ is reached. Any attempt of CPMG at this regime did not yield any improvement of the coherence time. This indicates rational synthetic routes that can be used to improve the room- $T$ coherence or integration into devices, e.g. via imprisonment into matrices such as zeolites and porous membranes or into liquid crystals. These results also open the path to the use of disperse graphenoids for sensing applications [65] in various environments [66-68], and can applications in bio-sciences and nanotechnology seem particularly interesting, also considering the hydrophobic and amphiphilic properties of biological membranes. 


\section{ACKNOWLEDGMENTS}

This work was financed by the Royal Society (URF and Research Grant), EPSRC (fellowship program EP/L011972/1), the European Research Coun- cil (ERC-CoG-773048-MMGNRs, ERC-CoG-819698T2DCP, Graphene Flagship-Core2-696656, and European Social Fund), German DFG (Excellence Cluster CFAED and EnhanceNano-391979941) and Saxony ESFProject-GRAPHD.
[1] M. A. Nielsen and I. Chuang, Quantum computation and quantum information (AAPT, 2002).

[2] T. D. Ladd, F. Jelezko, R. Laflamme, Y. Nakamura, C. Monroe, and J. L. OBrien, Nature 464, 45 (2010).

[3] N. V. Prokof'ev and P. C. E. Stamp, Rep. Prog. Phys. 63, 669 (2000).

[4] L. Viola, E. Knill, and S. Lloyd, Phys. Rev. Lett. 82, 2417 (1999).

[5] H. Bluhm, S. Foletti, I. Neder, M. Rudner, D. Mahalu, V. Umansky, and A. Yacoby, Nat. Phys. 7, 109 (2011).

[6] T. Van Der Sar, Z. H. Wang, M. S. Blok, H. Bernien, T. H. Taminiau, D. M. Toyli, D. A. Lidar, D. D. Awschalom, R. Hanson, and V. V. Dobrovitski, Nature 484, 82 (2012).

[7] N. Bar-Gill, L. M. Pham, C. Belthangady, D. Le Sage, P. Cappellaro, J. R. Maze, M. D. Lukin, A. Yacoby, and R. Walsworth, Nat. Commun. 3, 856 (2012).

[8] H. S. Knowles, D. M. Kara, and M. Atatüre, Phys. Rev. B 96, 1 (2017).

[9] M. H. Abobeih, J. Cramer, M. A. Bakker, N. Kalb, M. Markham, D. J. Twitchen, and T. H. Taminiau, Nat. Commun. 9, 1 (2018).

[10] C. E. Bradley, J. Randall, M. H. Abobeih, R. C. Berrevoets, M. J. Degen, M. A. Bakker, M. Markham, D. J. Twitchen, and T. H. Taminiau, Phys. Rev. X 9, 31045 (2019).

[11] G. Q. Liu, J. Xing, W. L. Ma, P. Wang, C. H. Li, H. C. Po, Y. R. Zhang, H. Fan, R. B. Liu, and X. Y. Pan, Phys. Rev. Lett. 118, 1 (2017).

[12] M. Atatüre, D. Englund, N. Vamivakas, S. Y. Lee, and J. Wrachtrup, Nat. Rev. Mat. 3, 38 (2018).

[13] D. D. Awschalom, R. Hanson, J. Wrachtrup, and B. B. Zhou, Nat. Phot. 12, 516 (2018).

[14] L. Bogani, Journal of Applied Physics 109, 07B115 (2011).

[15] S. Jiang, K. Goß, C. Cervetti, and L. Bogani, Science China Chemistry 55, 867 (2012).

[16] S.-D. Jiang, D. Maganas, N. Levesanos, E. Ferentinos, S. Haas, K. Thirunavukkuarasu, J. Krzystek, M. Dressel, L. Bogani, F. Neese, et al., Journal of the American Chemical Society 137, 12923 (2015).

[17] R. Moroni, R. Buzio, A. Chincarini, U. Valbusa, F. B. de Mongeot, L. Bogani, A. Caneschi, R. Sessoli, L. Cavigli, and M. Gurioli, Journal of Materials Chemistry 18, 109 (2008).

[18] R. Chen, R.-Q. Lu, P.-C. Shi, and X.-Y. Cao, Chinese Chemical Letters 27, 1175 (2016).

[19] A. Ardavan, O. Rival, J. J. L. Morton, S. J. Blundell, A. M. Tyryshkin, G. A. Timco, and R. E. P. Winpenny, Phys. Rev. Lett. 98, 057201 (2007).

[20] J. M. Zadrozny, J. Niklas, O. G. Poluektov, and D. E. Freedman, ACS Central Science 1, 488 (2015).

[21] C.-J. Yu, M. J. Graham, J. M. Zadrozny, J. Niklas, M. D. Krzyaniak, M. R. Wasielewski, O. G. Poluektov, and D. E. Freedman, Journal of the American Chemical So- ciety 138, 14678 (2016).

[22] M. Shiddiq, D. Komijani, Y. Duan, A. Gaita-Ariño, E. Coronado, and S. Hill, Nature 531, 348 (2016).

[23] J. M. Zadrozny, A. T. Gallagher, T. D. Harris, and D. E. Freedman, Journal of the American Chemical Society 139, 7089 (2017).

[24] A. Gaita-Ariño, F. Luis, S. Hill, and E. Coronado, Nature Chemistry 11, 301 (2019).

[25] J. M. Clemente-Juan, E. Coronado, and A. Gaita-Ariño, Chemical Society Reviews 41, 7464 (2012).

[26] E. Coronado, Nature Reviews Materials , 1 (2019).

[27] B. Trauzettel, D. V. Bulaev, D. Loss, and G. Burkard, Nat. Phys. 3, 192 (2007).

[28] M. Slota, A. Keerthi, W. K. Myers, E. Tretyakov, M. Baumgarten, A. Ardavan, H. Sadeghi, C. J. Lambert, A. Narita, K. Müllen, et al., Nature 557, 691 (2018).

[29] S. Mishra, D. Beyer, K. Eimre, S. Kezilebieke, R. Berger, O. Gröning, C. A. Pignedoli, K. Müllen, P. Liljeroth, P. Ruffieux, et al., Nature Nanotechnology , 1 (2019).

[30] E. A. Laird, F. Pei, and L. P. Kouwenhoven, Nat. Nanotechnol. 8, 565 (2013).

[31] F. Lombardi, A. Lodi, J. Ma, J. Liu, M. Slota, A. Narita, W. K. Myers, K. Müllen, X. Feng, and L. Bogani, Science 366, 1107 (2019).

[32] G. Balasubramanian, P. Neumann, D. Twitchen, M. Markham, R. Kolesov, N. Mizuochi, J. Isoya, J. Achard, J. Beck, J. Tissler, V. Jacques, P. R. Hemmer, F. Jelezko, and J. Wrachtrup, Nat. Mater. 8, 383 (2009).

[33] H. Y. Carr and E. M. Purcell, Physical review 94, 630 (1954).

[34] S. Meiboom and D. Gill, Rev. Sci. Instrum. 29, 688 (1958).

[35] A. Narita, X.-Y. Wang, X. Feng, and K. Müllen, Chemical Society Reviews 44, 6616 (2015).

[36] J. Ma, J. Liu, M. Baumgarten, Y. Fu, Y. Z. Tan, K. S. Schellhammer, F. Ortmann, G. Cuniberti, H. Komber, R. Berger, K. Müllen, and X. Feng, Angew. Chemie Int. Ed. 56, 3280 (2017).

[37] Y. G. Tullimi, W. Zeng, X. Lu, and J. Wu, Chem. Commun. 54, 2186 (2018).

[38] Y. Ni, T. Y. Gopalakrishna, H. Phan, T. S. Herng, S. Wu, Y. Han, J. Ding, and J. Wu, Angewandte Chemie International Edition 57, 9697 (2018).

[39] C. Liu, Y. Ni, X. Lu, G. Li, and J. Wu, Accounts of chemical research 52, 2309 (2019).

[40] A. Schweiger and G. Jeschke, Principles of Pulse Electron Paramagnetic Resonance (Oxford University Press, 2001).

[41] S. Stoll and A. Schweiger, J. Magn. Reson. 178, 42 (2006).

[42] K. Khodjasteh and D. A. Lidar, Phys. Rev. Lett. 95, 1 (2005).

[43] O. Kern and G. Alber, Phys. Rev. Lett. 95, 250501 (2005). 
[44] G. S. Uhrig, Phys. Rev. Lett. 98, 7 (2007).

[45] D. Farfurnik, A. Jarmola, L. M. Pham, Z. H. Wang, V. V. Dobrovitski, R. L. Walsworth, D. Budker, and N. BarGill, Phys. Rev. B 92, 1 (2015).

[46] K. Khodjasteh and D. A. Lidar, Phys. Rev. A - At. Mol. Opt. Phys. 75, 1 (2007).

[47] G. de Lange, Z. H. Wang, D. Riste, V. V. Dobrovitski, and R. Hanson, Science 330, 60 (2010).

[48] N. Bar-Gill, L. M. Pham, A. Jarmola, D. Budker, and R. L. Walsworth, Nat. Commun. 4, 1743 (2013).

[49] G. A. Álvarez, A. Ajoy, X. Peng, and D. Suter, Phys. Rev. A - At. Mol. Opt. Phys. 82, 1 (2010).

[50] J. J. L. Morton, A. M. Tyryshkin, A. Ardavan, K. Porfyrakis, S. A. Lyon, and G. A. D. Briggs, Phys. Rev. A 71, 1 (2005).

[51] J. H. Shim, I. Niemeyer, J. Zhang, and D. Suter, Epl 99 (2012), 10.1209/0295-5075/99/40004.

[52] J. Soetbeer, M. Hülsmann, A. Godt, Y. Polyhach, and G. Jeschke, Phys. Chem. Chem. Phys. 20, 1615 (2018).

[53] M. A. A. Ahmed, G. A. Álvarez, and D. Suter, Physical Review A 87, 042309 (2013).

[54] Z.-H. Wang, G. De Lange, D. Ristè, R. Hanson, and V. Dobrovitski, Physical Review B 85, 155204 (2012).

[55] A. Dréau, P. Jamonneau, O. Gazzano, S. Kosen, J.-F. Roch, J. Maze, and V. Jacques, Physical review letters 113, 137601 (2014).

[56] A. M. Souza, G. A. Álvarez, and D. Suter, Philosophical Transactions of the Royal Society A: Mathematical, Physical and Engineering Sciences 370, 4748 (2012).

[57] J. E. Lang, T. Madhavan, J.-P. Tetienne, D. A. Broad- way, L. T. Hall, T. Teraji, T. S. Monteiro, A. Stacey, and L. C. L. Hollenberg, Phys. Rev. A 99, 012110 (2019).

[58] A. M. Souza, G. A. Ávarez, and D. Suter, Phys. Rev. Lett. 106, 1 (2011).

[59] J. Zhang, A. M. Souza, F. D. Brandao, and D. Suter, Phys. Rev. Lett. 112, 1 (2014).

[60] M. H. Levitt, Progress in Nuclear Magnetic Resonance Spectroscopy 18, 61 (1986).

[61] H. K. Cummins, G. Llewellyn, and J. A. Jones, Phys. Rev. A - At. Mol. Opt. Phys. 67, 7 (2003).

[62] J. J. L. Morton, A. M. Tyryshkin, A. Ardavan, K. Porfyrakis, S. A. Lyon, and G. A. D. Briggs, Phys. Rev. Lett. 95, 1 (2005).

[63] P. E. Spindler, P. Schöps, W. Kallies, S. J. Glaser, and T. F. Prisner, J. Magn. Reson. 280, 30 (2017).

[64] A. A. Maudsley, Journal of Magnetic Resonance (1969) 69, 488 (1986).

[65] D. Schmid-Lorch, T. Haberle, F. Reinhard, A. Zappe, M. Slota, L. Bogani, A. Finkler, and J. Wrachtrup, Nano letters 15, 4942 (2015).

[66] R. Konnerth, C. Cervetti, A. Narita, X. Feng, K. Müllen, A. Hoyer, M. Burghard, K. Kern, M. Dressel, and L. Bogani, Nanoscale 7, 12807 (2015).

[67] C. Cervetti, E. Heintze, and L. Bogani, Dalton transactions 43, 4220 (2014).

[68] M. Mergenthaler, J. Liu, J. J. Le Roy, N. Ares, A. L. Thompson, L. Bogani, F. Luis, S. J. Blundell, T. Lancaster, A. Ardavan, et al., Physical review letters 119, 147701 (2017). 